\title{
Dirofilariasis canina. Nuevas perspectivas diagnósticas y terapéuticas
}

Anderson Machado-Casas ${ }^{1} \otimes$

$1 \quad$ Especialista en Cardiología Clínica Universidad de Buenos Aires. Email: vetmachado11@gmail.com

La dirofilariasis canina y felina es una enfermedad endémica, altamente contagiosa, parasitaria, que según los últimos mapeos epidemiológicos está presente en prácticamente todo el territorio nacional, pero principalmente en regiones costeras. Se considera un padecimiento crónico con consecuencias reversibles cuando se diagnostica de forma adecuada y temprana. El retraso en el diagnóstico trae como consecuencia la instauración de hipertensión pulmonar o de síndromes hemolíticos fatales para el paciente.

El objetivo principal de esta ponencia es presentar los diferentes eventos fisiopatológicos que presenta un canino infectado con esta enfermedad, así como mostrar las particularidades diagnósticas de los estados de la dirofilariasis cardiorrespiratoria y las alternativas terapéuticas que tenemos los clínicos veterinarios ante esta patología.

Se realizará una demostración de cómo diagnosticar ecocardiográficamente la dirofilariasis y la hipertensión pulmonar que desarrollan los pacientes en estados medios y avanzados de la enfermedad y se mostrará los datos de seguimiento que se ha realizado los últimos tres años de los pacientes remitidos a nuestro centro veterinario.

Autor para correspondencia Anderson Machado-Casas: vetmachado11@gmail.com 